\title{
Exact Recursion Relation Approach to Spin-1 Two-Leg Ladder
}

\author{
E. AlbayraK* \\ Erciyes University, Department of Physics, 38039, Kayseri, Turkey \\ Received: 28.07.2021 \& Accepted: 23.09.2021 \\ Doi: $10.12693 /$ APhysPolA.140.273 \\ *e-mail: albayrak@erciyes.edu.tr
}

\begin{abstract}
A two-leg ladder spin-1 model is constructed and examined in terms of the exact recursion relations. The model consists of two interchain and one intrachain bilinear exchange interaction parameters $J$ in addition to the single ion anisotropy parameter $D$ and external magnetic field $H$. Both the ferromagnetic, $J>0$, favoring the parallel alignment between the nearest-neighbor spins and antiferromagnetic, $J<0$, for antiparallel alignment of the nearest-neighbor spins are considered. The thermal and external magnetic field variations of magnetizations and susceptibilities belonging to each chain are examined. Different regions with ferromagnetic, ferrimagnetic, antiferromagnetic, non-magnetic, or chaotic phases are observed. Magnetization plateaus and more than one susceptibility peak are also found.
\end{abstract}

topics: Ising ladder, spin-1, plateaus, susceptibility

\section{Introduction}

Low-dimensional spin systems with competing exchange interactions, $J>0$, favoring ferromagnetic (FM) phase, and $J<0$, favoring antiferromagnetic $(\mathrm{AFM})$ phase, between the spins of given topology may lead to very high frustrations. Magnetic ladders are such systems which are mainly studied in connection with spin liquids [1], superconductivity [2], thermal conductivity [3], dimerized ground state [4], low temperature magnetization with plateaus and jumps [5], etc. It is known that there are some materials such as cuprates $\mathrm{Sr}_{n-1} \mathrm{Cu}_{n+1} \mathrm{O}_{2 n}, \mathrm{Sr}_{1-n} \mathrm{Cu}_{n} \mathrm{O}_{2}$, and $\mathrm{La}_{4+4 n} \mathrm{Cu}_{8+2 n} \mathrm{O}_{14+8 n}$ which consist of ladder structures [6-11]. The experimental properties of the materials such as $\mathrm{SrCu}_{2} \mathrm{O}_{3}, \mathrm{La}_{6} \mathrm{Ca}_{8} \mathrm{Cu}_{24} \mathrm{O}_{41}$, and $\mathrm{Cu}_{2}\left(\mathrm{C}_{5} \mathrm{H}_{12} \mathrm{~N}_{2}\right)_{2} \mathrm{Cl}_{4}(\mathrm{CuHpCl})$ can be analyzed by a two-leg Heisenberg antiferromagnet [12-14].

Theoretically, the Ising or Heisenberg two-leg ladders with different topology of interactions between the spins of a given system have also received the scientists' attention. The ground state properties of a one-dimensional Ising chain with the nearestneighbor (NN) FM interaction $J_{l}$, and a $k$-th neighbor AFM interaction $J_{k}$ were considered [15]. The Ising spin ladder with interchain and intrachain couplings was studied to investigate its thermal behaviors including specific heat [16]. The Ising approximation was proposed for the Heisenberg model with two- and three-spin exchange interactions on a zigzag ladder and examined by using the transfer matrix technique [17]. The mixed spin- $\frac{1}{2}$ and $\operatorname{spin}-S\left(S>\frac{1}{2}\right)$ Ising model on a rope ladder was examined by using the decoration-iteration mapping transformation [18]. The magnetocaloric properties of the two-leg frustrated spin ladder system were investigated by the use of the transfer matrix method [19]. The ground states of frustrated spin-1 Ising-Heisenberg two-leg ladder were rigorously found by taking the advantage of local conservation of the total spin on each rung [20]. The mixed spin- $\left(\frac{3}{2}, 1\right)$ ladder system with the AFM rung coupling and the next NN interaction was exactly solved to investigate its ground state and thermodynamic properties [21]. The spin- $\frac{1}{2}$ Ising-Heisenberg two-leg ladder accounting for alternating Ising and Heisenberg inter-leg couplings was rigorously mapped onto the mixed spin- $\left(\frac{3}{2}, \frac{1}{2}\right)$ IsingHeisenberg diamond chain [22]. Two types of the Ising-Heisenberg ladders consisting of spin- $\frac{1}{2}$ particles partitioned into adjoined block formed from butterfly-shaped plaquettes were investigated by the transfer matrix approach [23]. The dynamic magnetic behaviors of a ferrimagnetic (FI) mixed spin- $\left(1, \frac{3}{2}\right)$ Ising ladder-type graphene nanoribbon in a time-dependent magnetic field were studied by utilizing the Monte Carlo (MC) simulation [24] and similarly the effects of the anisotropies, exchange couplings, longitudinal magnetic field, and temperature on the magnetic and thermodynamic properties were also considered [25]. 


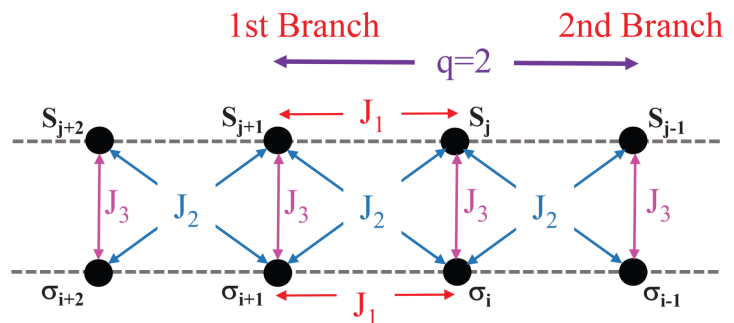

Fig. 1. Schematic diagram of a two-leg ladder: bilinear interaction parameter $J_{1}$ is along the $\mathrm{NN}$ spins of each chain, $J_{2}$ is between the spins of chains at cross positions, $J_{3}$ is along the spins of each rung, $D$ is the crystal field and $H$ is the external magnetic field at each site, with $\sigma_{i}$ and $S_{j}$ indicating the spins of upper and lower chains. Two branches of the ladder are also shown for which $q=2$.

It should be mentioned that one-dimensional classical short-range models in statistical physics are proven to be free of the order-disorder phase transitions, i.e., when the magnetizations become zero separating the FM and paramagnetic phases. The general nonexistence theorem for phase transitions in one-dimensional systems with short-range interactions was examined and the physical examples of such transitions were given [26]. The failure of the effective field theory in predicting a false spontaneous long-range order and phase transition of the Ising nanoparticles, nanoislands, nanotubes, and nanowires with either zero- or one-dimensional magnetic dimensionality was clarified [27]. In addition, the necessary conditions for the existence of a phase transition in a one-dimensional Ising ferromagnet were proven [28].

In this work, a two-leg ladder spin-1 Ising model is studied in terms of exact recursion relations (ERR), applied for the first time to the best of our knowledge, with two interchain and one intrachain bilinear exchange interaction parameters, single-ion anisotropy, and external magnetic field. The thermal and $H$ variations of magnetizations and susceptibilities are examined in terms of $J_{\mathrm{S}}$ and $D$. Very interesting results in agreement with the literature are found.

\section{Formulation}

The Hamiltonian of the two-leg ladder model includes the bilinear interaction parameter $J_{1}$ along the NN spins of each chain, the parameter between the spins of chains at cross positions $J_{2}$, the parameter $J_{3}$ along the spins of each rung, the crystal field $D$ and the external magnetic field $H$ acting to each spin site. The Hamiltonian may be written as

$$
\begin{aligned}
\hat{\mathcal{H}} & =-J_{1} \sum_{<i, i+1>} \sigma_{i} \sigma_{i+1}-J_{1} \sum_{\langle j, j+1\rangle} S_{j} S_{j+1} \\
& -J_{2} \sum_{\langle i, j+1\rangle} \sigma_{i} S_{j+1}-J_{2} \sum_{\langle i+1, j\rangle} \sigma_{i+1} S_{j} \\
& -J_{3} \sum_{\langle i, j\rangle} \sigma_{i} S_{j}-D \sum_{i} \sigma_{i}^{2}-D \sum_{j} S_{j}^{2} \\
& -H \sum_{i} \sigma_{i}-H \sum_{j} S_{j},
\end{aligned}
$$

where $\sigma_{i}$ and $S_{j}$ denote the spins of upper and lower chains assumed to be spin- 1 with the values \pm 1 and 0 while $\langle i, j\rangle$ indicate the summation along the given direction as displayed in Fig. 1 . The $J_{\mathrm{S}}$ can be positive or negative corresponding to the FM and AFM phases, respectively.

In order to calculate the magnetizations belonging to upper and lower chains, i.e., $M_{\sigma}$ and $M_{S}$, a similar exact ERR approach of the Bethe lattice case is employed [29-32]. It should be mentioned that the ERR approach can be applied easily to any spin system giving results better than the mean field approaches since it also considers the internal structure of the given spin configuration via the coordination number. First, one takes the $i$-th and $j$-th spins of the chains along the 0th rung or the central rung. Each rung on the ladder has always two NN rungs.

Let us start with the partition function

$$
Z=\sum_{\mathrm{Spc}} \mathrm{e}^{-\beta \hat{\mathcal{H}}}=\sum_{\mathrm{Spc}} P(\mathrm{Spc})
$$

where Spc refers to the spin configuration, while $\beta=1 / k_{\mathrm{B}} T$ and the Boltzmann constant $k_{\mathrm{B}}$ is set to 1.0. Now, if the central rung is isolated from the others, i.e., this rung is cut from the others, one gets an unnormalized probability distribution $P\left(\sigma_{i}, S_{j}\right)$ given as

$$
\begin{aligned}
& P\left(\sigma_{i}, S_{j}\right)=\mathrm{e}^{\beta J_{3} \sigma_{i} S_{j}+\beta D\left(\sigma_{i}^{2}+S_{j}^{2}\right)+\beta H\left(\sigma_{i}+S_{j}\right)} \\
& \quad \times \prod_{k=1}^{q=2} Q_{n}\left(\sigma_{i}, S_{j} \mid\left\{\sigma_{i+1}, S_{j+1}\right\}^{k}\right),
\end{aligned}
$$

where $Q_{n}$ includes the rest of the interactions on the ladder, $q=2$ is the number of the NN rungs and $k$ refers to the branch of the ladder on which one traverses along the ladder. If now the ladder is cut at the first rung with the spins $\sigma_{i+1}$ and $S_{j+1}$ one gets

$$
\begin{aligned}
& Q_{n}\left(\sigma_{i}, S_{j} \mid \sigma_{i+1}, S_{j+1}\right)=\exp \left(\beta J_{1}\left(\sigma_{i} \sigma_{i+1}+S_{j} S_{j+1}\right)+\beta J_{2}\left(\sigma_{i+1} S_{j}+\sigma_{i} S_{j+1}\right)+\beta J_{3} \sigma_{i+1} S_{j+1}\right) \\
& \quad \times \exp \left(\beta D\left(\sigma_{i+1}^{2}+S_{j+1}^{2}\right)+\beta H\left(\sigma_{i+1}+S_{j+1}\right)\right) \prod_{l=1}^{q-1} Q_{n-1}\left(\sigma_{i+1}, S_{j+1} \mid\left\{\sigma_{i+2}, S_{j+2}\right\}^{l}\right) .
\end{aligned}
$$


In order to calculate the ERRs for given spin values, \pm 1 and 0 , along the ladder, the first one introduces a function of the form

$$
g_{n}\left(\sigma_{i}, S_{j}\right)=\sum_{\substack{\sigma_{i+1} \\ S_{j+1}}} Q_{n}\left(\sigma_{i}, S_{j} \mid \sigma_{i+1}, S_{j+1}\right) .
$$

Together with (4), it reads as

$$
\begin{aligned}
& g_{n}\left(\sigma_{i}, S_{j}\right)=\sum_{\substack{\sigma_{i+1} \\
S_{j+1}}} \exp \left(\beta J_{1}\left(\sigma_{i} \sigma_{i+1}+S_{j} S_{j+1}\right)\right) \\
& \quad \times \exp \left(\beta J_{2}\left(\sigma_{i+1} S_{j}+\sigma_{i} S_{j+1}\right)+\beta J_{3} \sigma_{i+1} S_{j+1}\right) \\
& \quad \times \exp \left(\beta D\left(\sigma_{i+1}^{2}+S_{j+1}^{2}\right)+\beta H\left(\sigma_{i+1}+S_{j+1}\right)\right) \\
& \quad \times\left(g_{n-1}\left(\sigma_{i+1}, S_{j+1}\right)\right)^{p},
\end{aligned}
$$

where $p=q-1$. As a result, one gets nine $g_{n}$ functions as follows:

(i) for $\sigma_{i}=+1, S_{j}=+1$,

$$
\begin{aligned}
& g_{n}(+,+)=\mathrm{e}^{\beta\left(2 D+2 H+2 J_{1}+2 J_{2}+J_{3}\right)}\left[g_{n-1}(+,+)\right]^{p} \\
& \quad+\mathrm{e}^{\beta\left(D+H+J_{1}+J_{2}\right)}\left[g_{n-1}(+, 0)\right]^{p} \\
& +\mathrm{e}^{\beta\left(2 D-J_{3}\right)}\left[g_{n-1}(+,-)\right]^{p} \\
& \quad+\mathrm{e}^{\beta\left(D+H+J_{1}+J_{2}\right)}\left[g_{n-1}(0,+)\right]^{p}+\left(g_{n-1}(0,0)\right)^{p} \\
& +\mathrm{e}^{\beta\left(D-H-J_{1}-J_{2}\right)}\left[g_{n-1}(0,-)\right]^{p} \\
& +\mathrm{e}^{\beta\left(2 D-J_{3}\right)}\left[g_{n-1}(-,+)\right]^{p} \\
& +\mathrm{e}^{\beta\left(D-H-J_{1}-J_{2}\right)}\left[g_{n-1}(-, 0)\right]^{p} \\
& +\mathrm{e}^{\beta\left(2 D-2 H-2 J_{1}-2 J_{2}+J_{3}\right)}\left[g_{n-1}(-,-)\right]^{p},
\end{aligned}
$$

(ii) for $\sigma_{i}=+1, S_{j}=0$,

$$
\begin{aligned}
& g_{n}(+, 0)=\mathrm{e}^{\beta\left(2 D+2 H+J_{1}+J_{2}+J_{3}\right)}\left[g_{n-1}(+,+)\right]^{p} \\
& \quad+\mathrm{e}^{\beta\left(D+H+J_{1}\right)}\left[g_{n-1}(+, 0)\right]^{p} \\
& +\mathrm{e}^{\beta\left(2 D+J_{1}-J_{2}-J_{3}\right)}\left[g_{n-1}(+,-)\right]^{p} \\
& ++\mathrm{e}^{\beta\left(D+H+J_{2}\right)}\left[g_{n-1}(0,+)\right]^{p}+\left[g_{n-1}(0,0)\right]^{p} \\
& +\mathrm{e}^{\beta\left(D-H-J_{2}\right)}\left[g_{n-1}(0,-)\right]^{p} \\
& ++\mathrm{e}^{\beta\left(2 D-J_{1}+J_{2}-J_{3}\right)}\left[g_{n-1}(-,+)\right]^{p} \\
& +\mathrm{e}^{\beta\left(D-H-J_{1}\right)}\left[g_{n-1}(-, 0)\right]^{p} \\
& +\mathrm{e}^{\beta\left(2 D-2 H-J_{1}-J_{2}+J_{3}\right)}\left[g_{n-1}(-,-)\right]^{p}
\end{aligned}
$$

(iii) for $\sigma_{i}=+1, S_{j}=-1$,

$$
\begin{aligned}
& g_{n}(+,-)=\mathrm{e}^{\beta\left(2 D+2 H+J_{3}\right)}\left[g_{n-1}(+,+)\right]^{p} \\
& \quad+\mathrm{e}^{\beta\left(D+H+J_{1}-J_{2}\right)}\left[g_{n-1}(+, 0)\right]^{p} \\
& +\mathrm{e}^{\beta\left(2 D+2 J_{1}-2 J_{2}-J_{3}\right)}\left[g_{n-1}(+,-)\right]^{p} \\
& +\mathrm{e}^{\beta\left(D+H-J_{1}+J_{2}\right)}\left[g_{n-1}(0,+)\right]^{p}+\left[g_{n-1}(0,0)\right]^{p} \\
& +\mathrm{e}^{\beta\left(D-H+J_{1}-J_{2}\right)}\left[g_{n-1}(0,-)\right]^{p} \\
& +\mathrm{e}^{\beta\left(2 D-2 J_{1}+2 J_{2}-J_{3}\right)}\left[g_{n-1}(-,+)\right]^{p} \\
& +\mathrm{e}^{\beta\left(D-H-J_{1}+J_{2}\right)}\left[g_{n-1}(-, 0)\right]^{p} \\
& +\mathrm{e}^{\beta\left(2 D-2 H+J_{3}\right)}\left[g_{n-1}(-,-)\right]^{p},
\end{aligned}
$$

(iv) for $\sigma_{i}=0, S_{j}=+1$,

$$
\begin{aligned}
& g_{n}(0,+)=\mathrm{e}^{\beta\left(2 D+2 H+J_{1}+J_{2}+J_{3}\right)}\left[g_{n-1}(+,+)\right]^{p} \\
& +\mathrm{e}^{\beta\left(D+H+J_{2}\right)}\left[g_{n-1}(+, 0)\right]^{p} \\
& +\mathrm{e}^{\beta\left(2 D-J_{1}+J_{2}-J_{3}\right)}\left[g_{n-1}(+,-)\right]^{p} \\
& +\mathrm{e}^{\beta\left(D+H+J_{1}\right)}\left[g_{n-1}(0,+)\right]^{p}+\left[g_{n-1}(0,0)\right]^{p} \\
& +\mathrm{e}^{\beta\left(D-H-J_{1}\right)}\left[g_{n-1}(0,-)\right]^{p} \\
& +\mathrm{e}^{\beta\left(2 D+J_{1}-J_{2}-J_{3}\right)}\left[g_{n-1}(-,+)\right]^{p} \\
& +\mathrm{e}^{\beta\left(D-H-J_{2}\right)}\left[g_{n-1}(-, 0)\right]^{p} \\
& +\mathrm{e}^{\beta\left(2 D-2 H-J_{1}-J_{2}+J_{3}\right)}\left[g_{n-1}(-,-)\right]^{p},
\end{aligned}
$$

(v) for $\sigma_{i}=0, S_{j}=0$,

$$
\begin{aligned}
& g_{n}(0,0)=\mathrm{e}^{\beta\left(2 D+2 H+J_{3}\right)}\left[g_{n-1}(+,+)\right]^{p} \\
& +\mathrm{e}^{\beta(D+H)}\left[g_{n-1}(+, 0)\right]^{p} \\
& +\mathrm{e}^{\beta\left(2 D-J_{3}\right)}\left[g_{n-1}(+,-)\right]^{p} \\
& +\mathrm{e}^{\beta(D+H)}\left[g_{n-1}(0,+)\right]^{p}+\left[g_{n-1}(0,0)\right]^{p} \\
& +\mathrm{e}^{\beta(D-H)}\left[g_{n-1}(0,-)\right]^{p} \\
& +\mathrm{e}^{\beta\left(2 D-J_{3}\right)}\left[g_{n-1}(-,+)\right]^{p} \\
& +\mathrm{e}^{\beta(D-H)}\left[g_{n-1}(-, 0)\right]^{p} \\
& +\mathrm{e}^{\beta\left(2 D-2 H+J_{3}\right)}\left[g_{n-1}(-,-)\right]^{p},
\end{aligned}
$$

(vi) for $\sigma_{i}=0, S_{j}=-1$,

$$
\begin{aligned}
& g_{n}(0,-)=\mathrm{e}^{\beta\left(2 D+2 H-J_{1}-J_{2}+J_{3}\right)}\left[g_{n-1}(+,+)\right]^{p} \\
& +\mathrm{e}^{\beta\left(D+H-J_{2}\right)}\left[g_{n-1}(+, 0)\right]^{p} \\
& +\mathrm{e}^{\beta\left(2 D+J_{1}-J_{2}-J_{3}\right)}\left[g_{n-1}(+,-)\right]^{p} \\
& +\mathrm{e}^{\beta\left(D+H-J_{1}\right)}\left[g_{n-1}(0,+)\right]^{p}+\left[g_{n-1}(0,0)\right]^{p} \\
& +\mathrm{e}^{\beta\left(D-H+J_{1}\right)}\left[g_{n-1}(0,-)\right]^{p} \\
& +\mathrm{e}^{\beta\left(2 D-J_{1}+J_{2}-J_{3}\right)}\left[g_{n-1}(-,+)\right]^{p} \\
& +\mathrm{e}^{\beta\left(D-H+J_{2}\right)}\left[g_{n-1}(-, 0)\right]^{p} \\
& +\mathrm{e}^{\beta\left(2 D-2 H+J_{1}+J_{2}+J_{3}\right)}\left[g_{n-1}(-,-)\right]^{p},
\end{aligned}
$$

(vii) for $\sigma_{i}=-1, S_{j}=+1$,

$$
\begin{aligned}
& g_{n}(-,+)=\mathrm{e}^{\beta\left(2 D+2 H+J_{3}\right)}\left[g_{n-1}(+,+)\right]^{p} \\
& +\mathrm{e}^{\beta\left(D+H-J_{1}+J_{2}\right)}\left[g_{n-1}(+, 0)\right]^{p} \\
& +\mathrm{e}^{\beta\left(2 D-2 J_{1}+2 J_{2}-J_{3}\right)}\left[g_{n-1}(+,-)\right]^{p} \\
& +\mathrm{e}^{\beta\left(D+H+J_{1}-J_{2}\right)}\left[g_{n-1}(0,+)\right]^{p}+\left[g_{n-1}(0,0)\right]^{p} \\
& +\mathrm{e}^{\beta\left(D-H-J_{1}+J_{2}\right)}\left[g_{n-1}(0,-)\right]^{p} \\
& +\mathrm{e}^{\beta\left(2 D+2 J_{1}-2 J_{2}-J_{3}\right)}\left[g_{n-1}(-,+)\right]^{p} \\
& +\mathrm{e}^{\beta\left(D-H+J_{1}-J_{2}\right)}\left[g_{n-1}(-, 0)\right]^{p} \\
& +\mathrm{e}^{\beta\left(2 D-2 H+J_{3}\right)}\left[g_{n-1}(-,-)\right]^{p}
\end{aligned}
$$


(viii) for $\sigma_{i}=-1, S_{j}=0$,

$$
\begin{aligned}
& g_{n}(-, 0)=\mathrm{e}^{\beta\left(2 D+2 H-J_{1}-J_{2}+J_{3}\right)}\left[g_{n-1}(+,+)\right]^{p} \\
& +\mathrm{e}^{\beta\left(D+H-J_{1}\right)}\left[g_{n-1}(+, 0)\right]^{p} \\
& +\mathrm{e}^{\beta\left(2 D-J_{1}+J_{2}-J_{3}\right)}\left[g_{n-1}(+,-)\right]^{p} \\
& +\mathrm{e}^{\beta\left(D+H-J_{2}\right)}\left[g_{n-1}(0,+)\right]^{p}+\left[g_{n-1}(0,0)\right]^{p} \\
& +\mathrm{e}^{\beta\left(D-H+J_{2}\right)}\left[g_{n-1}(0,-)\right]^{p} \\
& +\mathrm{e}^{\beta\left(2 D+J_{1}-J_{2}-J_{3}\right)}\left[g_{n-1}(-,+)\right]^{p} \\
& +\mathrm{e}^{\beta\left(D-H+J_{1}\right)}\left[g_{n-1}(-, 0)\right]^{p} \\
& +\mathrm{e}^{\beta\left(2 D-2 H+J_{1}+J_{2}+J_{3}\right)}\left[g_{n-1}(-,-)\right]^{p},
\end{aligned}
$$

(ix) for $\sigma_{i}=-1, S_{j}=-1$,

$$
\begin{aligned}
& g_{n}(-,-)=\mathrm{e}^{\beta\left(2 D+2 H-2 J_{1}-2 J_{2}+J_{3}\right)}\left[g_{n-1}(+,+)\right]^{p} \\
& \quad+\mathrm{e}^{\beta\left(D+H-J_{1}-J_{2}\right)}\left[g_{n-1}(+, 0)\right]^{p} \\
& \quad+\mathrm{e}^{\beta\left(2 D-J_{3}\right)}\left[g_{n-1}(+,-)\right]^{p} \\
& \quad+\mathrm{e}^{\beta\left(D+H-J_{1}-J_{2}\right)}\left[g_{n-1}(0,+)\right]^{p}+\left[g_{n-1}(0,0)\right]^{p} \\
& \quad+\mathrm{e}^{\beta\left(D-H+J_{1}+J_{2}\right)}\left[g_{n-1}(0,-)\right]^{p} \\
& +\mathrm{e}^{\beta\left(2 D-J_{3}\right)}\left[g_{n-1}(-,+)\right]^{p} \\
& +\mathrm{e}^{\beta\left(D-H+J_{1}+J_{2}\right)}\left[g_{n-1}(-, 0)\right]^{p} \\
& +\mathrm{e}^{\beta\left(2 D-2 H+2 J_{1}+2 J_{2}+J_{3}\right)}\left[g_{n-1}(-,-)\right]^{p} .
\end{aligned}
$$

Then, one can take the ratio of all the $g_{n}$ functions with any one of them, for example $g_{n}(-,-)$. Finally, one gets eight ERRs which behave like the equation of states and are found as

$$
\begin{aligned}
& X_{1}=\frac{g_{n}(+,+)}{g_{n}(-,-)}, \quad X_{2}=\frac{g_{n}(+, 0)}{g_{n}(-,-)}, \\
& X_{3}=\frac{g_{n}(+,-)}{g_{n}(-,-)}, \quad X_{4}=\frac{g_{n}(0,+)}{g_{n}(-,-)}, \\
& X_{5}=\frac{g_{n}(0,0)}{g_{n}(-,-)}, \quad X_{6}=\frac{g_{n}(0,-)}{g_{n}(-,-)}, \\
& X_{7}=\frac{g_{n}(-,+)}{g_{n}(-,-)}, \quad X_{8}=\frac{g_{n}(-, 0)}{g_{n}(-,-)} .
\end{aligned}
$$

The formula for the partition function can be obtained from (2) and (3) which yields

$Z=\sum_{\sigma_{i}, S_{j}} \mathrm{e}^{\beta\left[D\left(\sigma_{i}^{2}+S_{j}^{2}\right)+H\left(\sigma_{i}+S_{j}\right)+J_{3} \sigma_{i} S_{j}\right]}\left[g_{n}\left(\sigma_{i}, S_{j}\right)\right]^{q}$.

Carrying out the summation over the spin values, one gets $Z$ in terms of the $g_{n}$ functions as

$$
\begin{aligned}
Z & =\mathrm{e}^{\beta\left(2 D+J_{3}+2 H\right)}\left[g_{n}(+,+)\right]^{q} \\
& +\mathrm{e}^{\beta(D+H)}\left[g_{n}(+, 0)\right]^{q}+\mathrm{e}^{\beta\left(2 D-J_{3}\right)}\left[g_{n}(+,-)\right]^{q} \\
& +\mathrm{e}^{\beta(D+H)}\left[g_{n}(0,+)\right]^{q}+\left[g_{n}(0,0)\right]^{q} \\
& +\mathrm{e}^{\beta(D-H)}\left[g_{n}(0,-)\right]^{q}+\mathrm{e}^{\beta\left(2 D-J_{3}\right)}\left[g_{n}(-,+)\right]^{q} \\
& +\mathrm{e}^{\beta(D-H)}\left[g_{n}(-, 0)\right]^{q} \\
& +\mathrm{e}^{\beta\left(2 D+J_{3}-2 H\right)}\left[g_{n}(-,-)\right]^{q} .
\end{aligned}
$$

The magnetizations belonging to the upper and lower chains can be calculated from

$$
\begin{aligned}
& M_{\sigma}=\frac{1}{Z} \sum_{\sigma_{i}, S_{j}} \sigma_{i} P\left(\sigma_{i}, S_{j}\right)= \\
& \frac{1}{Z} \sum_{\sigma_{i}, S_{j}} \sigma_{i} \mathrm{e}^{\beta\left[D\left(\sigma_{i}^{2}+S_{j}^{2}\right)+H\left(\sigma_{i}+S_{j}\right)+J_{3} \sigma_{i} S_{j}\right]}\left[g_{n}\left(\sigma_{i}, S_{j}\right)\right]^{q}
\end{aligned}
$$

and

$$
\begin{aligned}
& M_{S}=\frac{1}{Z} \sum_{\sigma_{i}, S_{j}} S_{j} P\left(\sigma_{i}, S_{j}\right)= \\
& \frac{1}{Z} \sum_{\sigma_{i}, S_{j}} S_{j} \mathrm{e}^{\beta\left[D\left(\sigma_{i}^{2}+S_{j}^{2}\right)+H\left(\sigma_{i}+S_{j}\right)+J_{3} \sigma_{i} S_{j}\right]}\left[g_{n}\left(\sigma_{i}, S_{j}\right)\right]^{q},
\end{aligned}
$$

respectively. Finally, the magnetizations in terms of the ERRs are found as

$$
\begin{aligned}
& M_{\sigma}=\frac{1}{Z^{\prime}}\left[\mathrm{e}^{\beta\left(2 D+J_{3}+2 H\right)} X_{1}^{q}+\mathrm{e}^{\beta(D+H)} X_{2}^{q}\right. \\
& +\mathrm{e}^{\beta\left(2 D-J_{3}\right)} X_{3}^{q}-\mathrm{e}^{\beta\left(2 D-J_{3}\right)} X_{7}^{q} \\
& \left.-\mathrm{e}^{\beta(D-H)} X_{8}^{q}-\mathrm{e}^{\beta\left(2 D+J_{3}-2 H\right)}\right]
\end{aligned}
$$

and

$$
\begin{aligned}
& M_{S}=\frac{1}{Z^{\prime}}\left[\mathrm{e}^{\beta\left(2 D+J_{3}+2 H\right)} X_{1}^{q}-\mathrm{e}^{\beta\left(2 D-J_{3}\right)} X_{3}^{q}\right. \\
& +\mathrm{e}^{\beta(D+H)} X_{4}^{q}-\mathrm{e}^{\beta(D-H)} X_{6}^{q} \\
& \left.+\mathrm{e}^{\beta\left(2 D-J_{3}\right)} X_{7}^{q}-\mathrm{e}^{\beta\left(2 D+J_{3}-2 H\right)}\right],
\end{aligned}
$$

where $Z^{\prime}$ in terms of ERRs is given as

$$
\begin{aligned}
Z^{\prime} & =\mathrm{e}^{\beta\left(2 D+J_{3}+2 H\right)} X_{1}^{q}+\mathrm{e}^{\beta(D+H)} X_{2}^{q} \\
& +\mathrm{e}^{\beta\left(2 D-J_{3}\right)} X_{3}^{q}+\mathrm{e}^{\beta(D+H)} X_{4}^{q}+X_{5}^{q} \\
& +\mathrm{e}^{\beta(D-H)} X_{6}^{q}+\mathrm{e}^{\beta\left(2 D-J_{3}\right)} X_{7}^{q} \\
& +\mathrm{e}^{\beta(D-H)} X_{8}^{q}+\mathrm{e}^{\beta\left(2 D+J_{3}-2 H\right)} .
\end{aligned}
$$

Note that the quadrupolar moments belonging to each chain, i.e., $Q_{\sigma}$ and $Q_{S}$, can be obtained by simply taking all the signs of each term in $M_{\sigma}$ and $M_{S}$ to be positive, respectively.

The susceptibilities of each chain can be obtained from the well-known definitions given as

$$
\chi_{\sigma}=\lim _{H \rightarrow 0} \frac{\partial M_{\sigma}}{\partial H} \quad \text { and } \quad \chi_{S}=\lim _{H \rightarrow 0} \frac{\partial M_{S}}{\partial H},
$$

which are too long to be given explicitly here.

The numerical values of the ERRs are obtained by the application of simple iterations which are terminated after the ERRs reach their saturation values. Afterwards, they are used to study the thermal and external magnetic field variations of magnetizations and susceptibilities.

\section{Thermal and $H$ variations of order parameters}

The dependence of the order parameters on the given system variables are discussed in this section. The thermal variations of the order parameters are studied when $H$ is turned off and on and then the $H$ variations at given temperatures are investigated. 

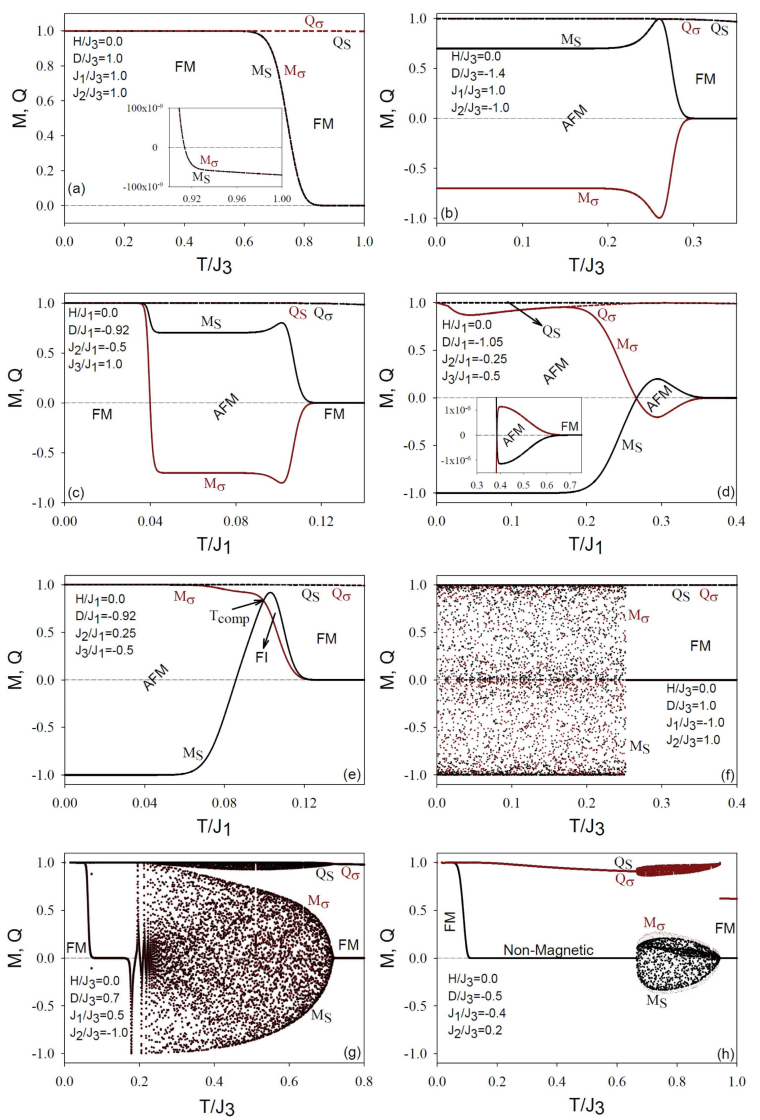

Fig. 2. Thermal variations of the order parameters with zero external magnetic field for: (a) $D / J_{3}=J_{1} / J_{3}=J_{2} / J_{3}=1.0$, (b) $D / J_{3}=-1.4$, $J_{1} / J_{3}=1.0, \quad J_{2} / J_{3}=-1.0, \quad$ (c) $D / J_{1}=-0.92$, $J_{2} / J_{1}=-0.5, \quad J_{3} / J_{1}=1.0, \quad(\mathrm{~d}) \quad D / J_{1}=-1.05$, $J_{2} / J_{1}=-0.25, J_{3} / J_{1}=-0.5$, (e) $D / J_{1}=-0.92$, $J_{2} / J_{1}=0.25, J_{3} / J_{1}=-0.5$, (f) $D / J_{3}=-J_{1} / J_{3}=$ $J_{2} / J_{3}=1.0, \quad(\mathrm{~g}) \quad D / J_{3}=0.7, \quad J_{1} / J_{3}=0.5$, $J_{2} / J_{3}=-1.0$, and (h) $D / J_{3}=-0.5, J_{1} / J_{3}=-0.4$, $J_{2} / J_{3}=0.2$.

In order to obtain different topological behaviors and to reduce the number of variables by one to ease the calculations, all the parameters are scaled by $J_{k}>0.0$ with $k$ taken to be 1 or 3 .

Let us first discuss the case with zero $H$, i.e., the spontaneous magnetizations and quadrupole moments. Figure $2 \mathrm{a}$ is obtained for $D / J_{3}=1.0$, $J_{1} / J_{3}=1.0$, and $J_{2} / J_{3}=1.0$. It shows the existence of an FM phase. One can see that the ground state value is 1.0 which decreases as the temperature increases, as expected. Thus, magnetizations decrease gradually as $T$ increases. After changing the sign, they become very small but never reach zero as seen in the inset, i.e., the FM phase persists even at high $T$ s. The nonexistence of phase transitions $[20,26]$ is also confirmed with this new approach.

Figure $2 \mathrm{~b}$ is, in turn, obtained for $D / J_{3}=-1.4$, $J_{1} / J_{3}=1.0$, and $J_{2} / J_{3}=-1.0$. It shows the AFM phase at lower $T$ s. Now, the ground state value is lowered to \pm 0.6999 for $M_{S}$ and $M_{\sigma}$, respectively, because of the competition between $J_{1}$ and $J_{2}$. As the temperature increases, magnetizations present peaks, then they drop closer to zero in a small temperature range and as $T$ increases further, they become vanishingly small presenting the FM phase. The increase of magnetizations as the temperature increases in the AFM phase is seen because the NN spins are antiparallel in the AFM phase but during the transition to the FM phase the spins start randomly orienting themselves which may lead to an increase in magnetizations (see a similar behavior in Fig. 2a and b of [25]).

Figure 2c is obtained for $D / J_{1}=-0.92$, $J_{2} / J_{1}=-0.5$, and $J_{3} / J_{1}=1.0$. First, it shows the FM phase, then the magnetizations are separated as $T$ increases with the sign change in $M_{\sigma}$, afterwards the phase is the AFM phase which finally goes into the FM phase region with very small magnetization values as before.

In Fig. $2 \mathrm{~d}$ for $D / J_{1}=-1.05, J_{2} / J_{1}=-0.25$, and $J_{3} / J_{1}=-0.5, M_{S}$ and $M_{\sigma}$ present the AFM phase. As $T$ increases, they are lowered to cross each other about zero temperature, then they change the sign but still exhibit the AFM phase. As seen in the inset, this behavior is repeated by the system one more time at very low magnetizations. As before, with a further increase of $T$, they become very small but do not reach zero at finite $T$ s.

Next, Fig. 2e is obtained for $D / J_{1}=-0.92$, $J_{2} / J_{1}=0.25$, and $J_{3} / J_{1}=-0.5$ which again present the AFM phase at low temperatures; as the temperature increases, both magnetizations decrease in magnitude, then $M_{S}$ changes the sign and crosses $M_{\sigma}$ at the compensation temperature $T_{\text {comp }}$. After the $T_{\text {comp }}$, the FI phase appears in a small temperature range. The rest is the same as before. It is very important to emphasize that the magnetizations in Fig. 2 can become very small (close to zero) at higher temperatures but never reach zero as if there is an external magnetic field acting on the system (see inset of Fig. 2a).

The next three figures are very interesting, since the order parameters move up and down randomly in some temperature range. This may be the sign of the existence of a spin glass (SG) phase which is not investigated further in this work. Figure $2 \mathrm{f}$ is obtained for $D / J_{3}=1.0, J_{1} / J_{3}=-1.0, J_{2} / J_{3}=1.0$ which shows this random behavior starting from zero temperature, i.e., at the ground state, and terminates as a knife cut at the FM border. In Fig. 2g, obtained for $D / J_{3}=0.7, J_{1} / J_{3}=0.5$, $J_{2} / J_{3}=-1.0$, the magnetizations start with the FM phase, then we see a few ups and downs and then the random behavior starts for both $M$ which again terminates at the FM phase border. It is interesting now that the borders of the random behavior region look like the magnetization lines seen in the AFM phase. Figure $2 \mathrm{~h}\left(D / J_{3}=-0.5\right.$, $\left.J_{1} / J_{3}=-0.4, J_{2} / J_{3}=0.2\right)$ is similar to the previous figure but now a non-magnetic region is seen 

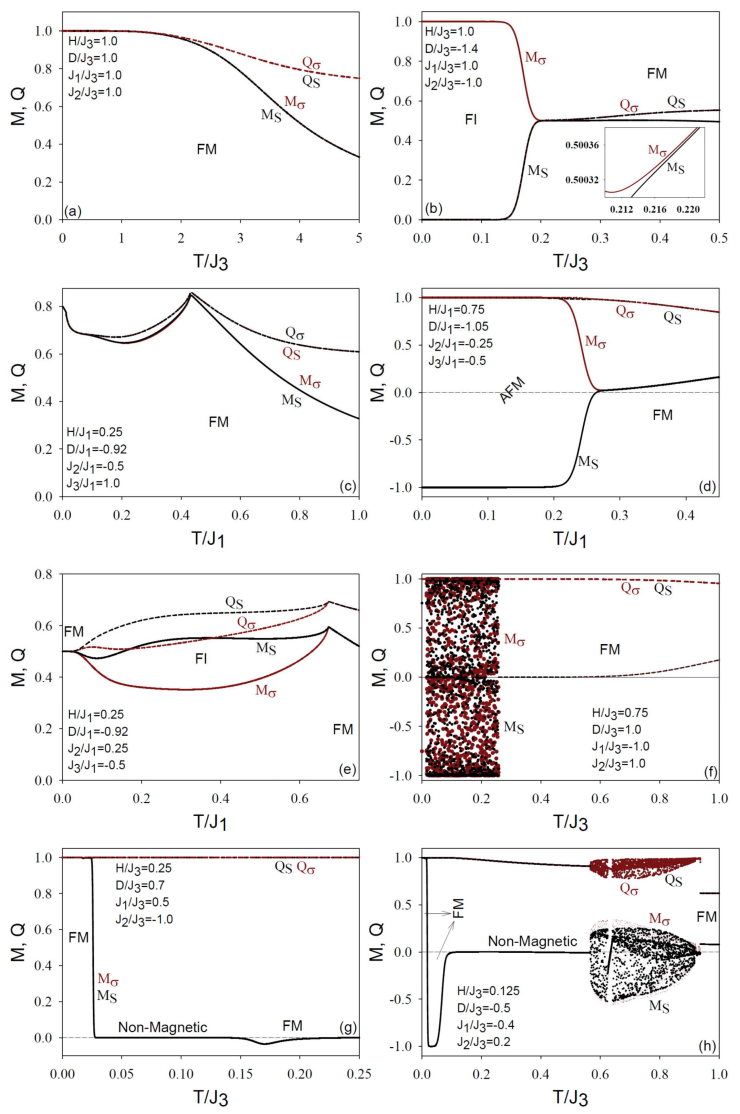

Fig. 3. Thermal variations of the order parameters with selected values of external magnetic field for: (a) $H / J_{3}=1.0$, (b) $H / J_{3}=1.0$, (c) $H / J_{1}=0.25$, (d) $H / J_{1}=0.75$, (e) $H / J_{1}=0.25$, (f) $H / J_{3}=0.750, \quad$ (g) $H / J_{3}=0.25, \quad$ and (h) $H / J_{3}=0.125$. The rest of the parameters, i.e., $D / J_{i}$ and $J_{i} / J_{k} \mathrm{~s}$, are the same, respectively, as the values of Fig. 2 .

in between the FM and the random region. The random region looks like a thumb print which again ends at the FM phase.

It is well known that the quadrupole moments make a little kink at the second-order phase transition temperatures. As seen in the figures, this kind of kink does not appear at all, which is also the sign of the non-existing finite temperature phase transitions in the model. Figure $2 \mathrm{~d}$ demonstrates that at low temperatures the $Q$ s are separated. Further, Fig. 2f shows that the $Q$ s are unaware of the random behavior of $M \mathrm{~s}$, in contrast to Fig. $2 \mathrm{~g}$ and $\mathrm{h}$.

Now let us see the results when $H$ is introduced into the model. It is well known that when there is an external magnetic field acting on the system, the order-disorder phase transitions are not seen. Figure 3a shows that all the order parameters start from 1.0 at zero temperature, then $M_{S}=M_{\sigma}$ and $Q_{S}=Q_{\sigma}$ decrease as $T$ increases. The FM phase is exhibited in this case. In Fig. $3 \mathrm{~b}, M_{S}=0.0$ and $M_{\sigma}=1.0$ at $T=0.0$, which indicates the FI phase. The quadrupole moments are equal to each other.
As seen in the inset, the magnetizations do not combine to give a phase transition. But they are very close to each other so the FM phase can be assumed. Figure 3c shows that the ground state value is about 0.8 which is other than the expected 0 and \pm 1 values. The order parameters first decrease and then increase as $T$ increases after presenting a peak at the same temperature, and afterwards they start decreasing. Again the system only presents the FM phase. Figure 3d illustrates the AFM phase at low temperatures and as $T$ increases, magnetizations decrease to approach each other about zero and subsequently they start increasing. Afterwards, the magnetizations become equal, i.e., the FM phase appears. The quadrupole moments present a little separation in the AFM phase region only, otherwise, they are equal to each other.

Figure 3e shows that all the lines start from 0.5 at $T=0.0$, i.e., the FM phase is present, then the $M$ lines are separated from where the FI phase appears. As $T$ increases, the magnetizations approach each other and follow each other very closely, so again the FM phase is seen. The quadrupole moments do the same thing but at higher $T$ s. Figure $3 \mathrm{f}$ is similar to Fig. 2f, but now after the random behavior terminates, the $M$ lines almost become zero and increase as the temperature increases. The $Q$ lines, however, decrease as $T$ increases. Again, the phase becomes the FM phase. As seen from Fig. 3g, all the lines start from 1.0 at zero temperature. The $M$ lines first exhibit the FM phase with a sharp drop close to zero, then there is a non-magnetic-like part, very close to zero, and finally the FM phase is seen again. Figure $3 \mathrm{~h}$ is similar to the previous figure but now the magnetization lines sharply drop from +1.0 to -1.0 and subsequently rise up very close to zero presenting double regions of the FM phase. Afterwards, as the temperature increases, first a non-magnetic region, and later the random behavior of the order parameters and finally the FM phase are seen again.

It should be noted that random behaviors of the order parameters have not been reported so far, but all the competitions between the system variables, namely $H$ and $J>0.0$ favors parallel alignment of spins, $J<0.0$ favors antiparallel alignment, $J_{1}, J_{2}$, and $J_{3}$ can be either positive or negative, and the negative values of the crystal field drive the system to the lowest spin value 0.0 , may induce this random behavior. Thus, this behavior deserves to get more attention with the other theoretical approaches for the probable existence of the SG phase.

Figure 4 involves the external magnetic field change of the order parameters at given temperatures. Figure $4 \mathrm{a}$ is obtained for $T / J_{3}=1.4$ and it shows that magnetizations start from -1.0 at negative $H$, then as $H$ decreases toward zero, it decreases and afterwards rises up to the value of 1.0 at higher $H \mathrm{~s}$ and quadrupole moments (the inset) present kinks close to the place where $M$ s change direction, i.e., about zero. Figure $4 \mathrm{~b}$ shows that the 

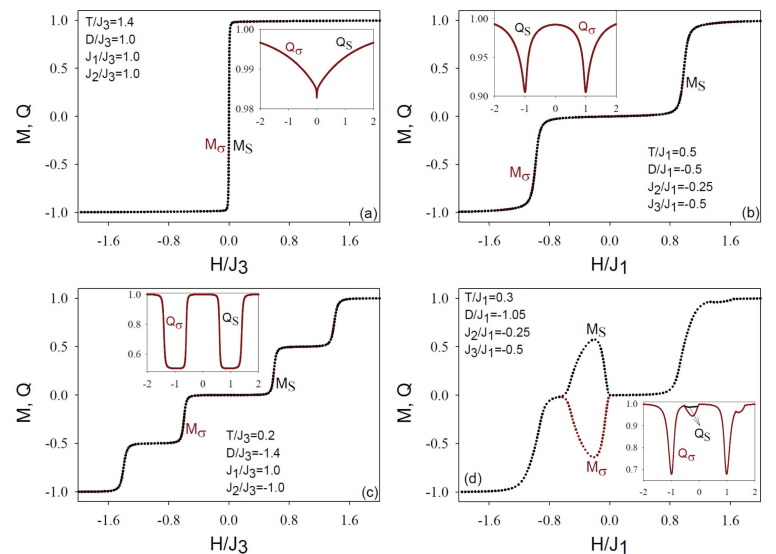

Fig. 4. External magnetic field variations of the order parameters for given temperatures: (a) $T / J_{3}=1.4, D / J_{3}=J_{1} / J_{3}=J_{2} / J_{3}=1.0$, (b) $T / J_{1}=0.5, \quad D / J_{1}=-0.5, \quad J_{2} / J_{1}=-0.25$, $J_{3} / J_{1}=-0.5, \quad$ (c) $\quad T / J_{3}=0.2, \quad D / J_{3}=-1.4$, $J_{1} / J_{3}=1.0, \quad J_{2} / J_{3}=-1.0$, and (d) $T / J_{1}=0.3$, $D / J_{1}=-1.05, J_{2} / J_{1}=-0.25, J_{3} / J_{1}=-0.5$.

magnetizations are of the Langevin type. The magnetization plateau appears at $M=0$ in the range around $-1.0 \leq H \leq 1.0$ and $M= \pm 1.0$, when about $H= \pm 1.0$, respectively, see also Fig. 3 of [17] for comparison. Again, quadrupole moments (the inset) present kinks at about $H= \pm 1.0$. As seen in Fig. 4c, the $M$ s start from -1.0 at $H=-2.0$, as $H$ increased the plateau at -1.0 , then they go down to -0.5 where there is another plateau at -0.5 , from where they go down to zero and another plateau at zero is observed. The same behavior is also observed for positive $H$ s. They are antisymmetric with respect to the $H$-axis, see a similar behavior in Fig. 4 of [17]. The quadrupole moments (the inset) start from 1.0, then go down to 0.5 presenting a plateau, subsequently go up to 1.0 at negative $H \mathrm{~s}$, and the same thing is repeated at positive $H$ s, i.e., it is symmetric with respect to the $H=0.0$-axis, as expected. Figure $4 \mathrm{~d}$ shows that both $M$ s start from -1.0 at $H=-2.0$, as $H$ increases, they decrease gradually to zero where they are separated, $M_{\sigma}$ makes a deep and $M_{S}$ makes a peak, forming a closed loop. Afterwards, they rise as $H$ increases but now no symmetry is observed. Both $Q$ (the inset) present kinks at about \pm 1.0 and also form a closed loop as $M$ s. Such a behavior has not been reported before elsewhere.

\section{Thermal and $H$ variations of susceptibilities}

The susceptibilities of each chain are obtained by using (24) which is calculated in the limit $H$ going to zero.

First, we present thermal variations of susceptibilities at $H=0$. Figure $5 \mathrm{a}$ is obtained for $D / J_{3}=J_{1} / J_{3}=J_{2} / J_{3}=1.0$ and shows only one
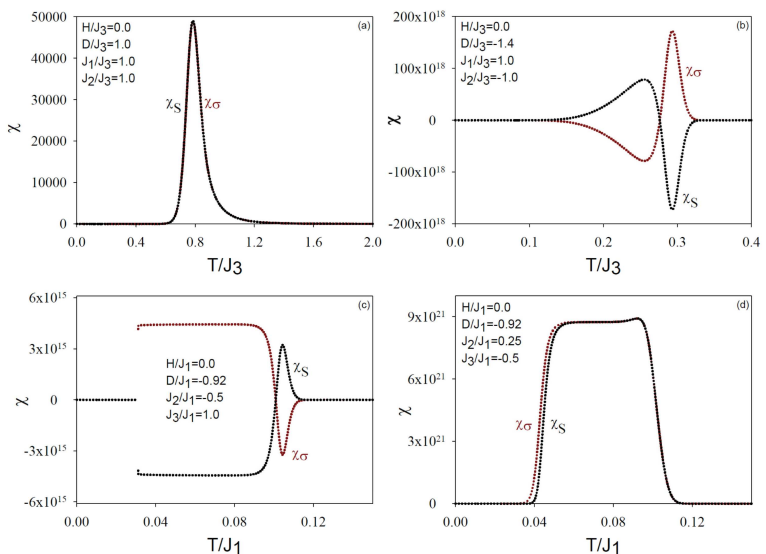

Fig. 5. Thermal variations of susceptibilities, $\chi_{\sigma}$ and $\chi_{S}$, with zero external magnetic field for: (a) $D / J_{3}=J_{1} / J_{3}=J_{2} / J_{3}=1.0$, (b) $D / J_{3}=-1.4$, $J_{1} / J_{3}=1.0, \quad J_{2} / J_{3}=-1.0, \quad$ (c) $D / J_{1}=-0.92$, $J_{2} / J_{1}=-0.5, J_{3} / J_{1}=1.0$, and (d) $D / J_{1}=-0.92$, $J_{2} / J_{1}=0.25, J_{3} / J_{1}=-0.5$.
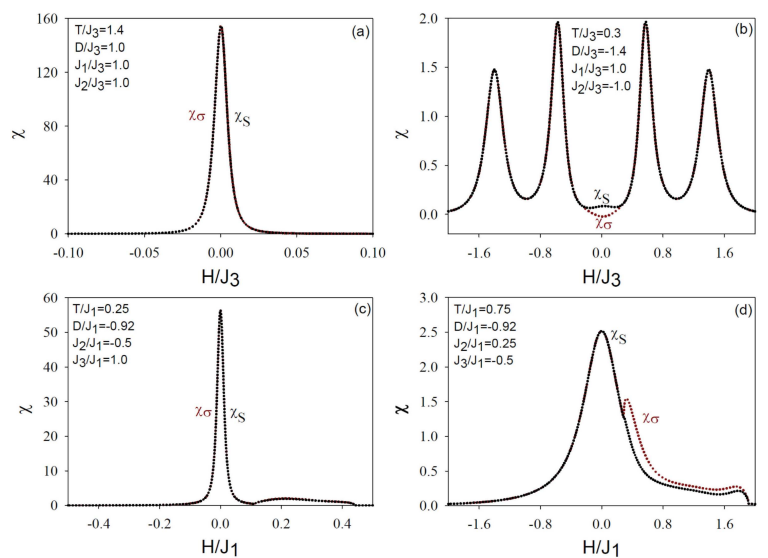

Fig. 6. External magnetic field variations of susceptibilities, $\chi_{\sigma}$ and $\chi_{S}$, for some selected temperatures: (a) $T / J_{3}=1.4, D / J_{3}=J_{1} / J_{3}=$ $J_{2} / J_{3}=1.0, \quad$ (b) $T / J_{3}=1.4, \quad D / J_{3}=-1.4$, $J_{1} / J_{3}=1.0, \quad J_{2} / J_{3}=-1.0, \quad$ (c) $T / J_{1}=0.25$, $D / J_{1}=-0.92, \quad J_{2} / J_{1}=-0.5, \quad J_{3} / J_{1}=1.0, \quad$ and (d) $T / J_{1}=0.75, \quad D / J_{1}=-0.92, \quad J_{2} / J_{1}=0.25$, $J_{3} / J_{1}=-0.5$.

peak in agreement with Fig. 2a. This peak is not sharp but instead it is broad so it does not indicate a phase transition. Figure 5b shows that the susceptibilities start from zero, then as $T$ increases, they are separated, $\chi_{S}$ rises up to a maximum and $\chi_{\sigma}$ goes down to a minimum and subsequently they turn back intersecting each other at zero with sign changes. After presenting peaks they go to zero. These two peaks are in agreement with Fig. 2b corresponding to rising up and going down of magnetizations. The two peaks in the susceptibilities are also observed in Fig. 4 of [21]. Figure 5c is similar to the previous one but it first presents a jump at very low temperatures and the first peak of Fig. 5b 
becomes a plateau in here, and then the second peak is seen. The jump at very low temperatures may not be trustworthy and may be the artifact of the iterations. In the last one, as shown in Fig. 5d, they start from zero, then go up to present a shallow region and subsequently go down to zero as $T$ is increased. They are separated at low $T$, and afterwards combine again forming a closed loop in agreement with the FI phase of Fig. 2e.

Finally, the $H$ variations of susceptibilities are presented at a given temperature. Figure 6 a shows a peak at $H=0.0$ in agreement with Figs. 2a-4a and Fig. 5a of [17]. Figure $6 \mathrm{~b}$ is calculated in correspondence with Fig. 4c, where two peaks for each region of $H$ are seen to be symmetric with respect to $H=0.0$ and each peak corresponds to the rise ups and downs in Fig. 4c (see also Fig. 4 of [17]). In the next two figures, the symmetries are spoiled again. Figure $6 \mathrm{c}$ shows a peak around zero $H$ and another peak at about 0.2 . Figure $6 \mathrm{~d}$ shows, in turn, that they start from zero at $H=-2.0$ and both follow the same path of rising up, presenting their common peaks at $H=0.0$, and then as $H$ increases, they both go down. The $\chi_{\text {sigma }}$ is separated, presenting a second peak forming a closed loop with $\chi_{S}$ in agreement with the FI phase of Figs. $2 \mathrm{~d}$ and $5 \mathrm{~d}$.

\section{Conclusions}

In this work, a two-leg ladder spin-1 Ising model was analyzed with a new approach in terms of exact recursion relations. It was found that the model exhibits the FM, AFM, FI, non-magnetic phases, and a random behavior resembling the SG phase. The latter has not been reported so far. The nonexistence of the finite temperature phase transitions is also supported with our findings. The thermal and external magnetic field change of the order parameters and susceptibilities are in agreement with the previous research as mentioned in the text. It should be noted that the free energy is not obtainable in the present approach, the free energy term has the coefficient $1 /(q-2)$ [29-32] which goes to infinity since each rung has two NN rungs, i.e., $q=2.0$. Therefore, the heat capacity is not obtained.

\section{References}

[1] A.F. Albuquerque, G.B. Martins, J. Phys. Condens. Matter 17, 2419 (2005).

[2] J. Akimitsu, Physica B 259, 1030 (1999).

[3] X. Zotos, Phys. Rev. Lett. 92, 067202 (2004).

[4] M.P. Gelfand, Phys. Rev. B 43, 8644 (1991).

[5] A. Honecker, F. Mila, M. Troyer, Eur. Phys. J. B 15, 227 (2000).

[6] T.M. Rice, S. Gopalan, M. Sigrist, Europhys. Lett. 23, 445 (1993).
[7] H.J. Schulz, Phys. Rev. B 34, 6372 (1986).

[8] I. Affleck, Phys. Rev. B 37, 5186 (1988).

[9] D.S. Rokhsar, S.A. Kivelson, Phys. Rev. Lett. 61, 2376 (1988).

[10] T.M. Rice, Z. Phys. B 103, 165 (1997).

[11] B.G. Levi, Phys. Today 49, 17 (1996).

[12] M. Azuma, Z. Hiroi, M. Takano, K. Ishida, Y. Kitaoka, Phys. Rev. Lett. 73, 3463 (1994).

[13] T. Imai, K.R. Thurber, K.M. Shen, A.W. Hunt, F.C. Chou, Phys. Rev. Lett. 81, 220 (1998).

[14] G. Chaboussant, P.A. Crowell, L.P. Lévy, O. Piovesana, A. Madouri, D. Mailly, Phys. Rev. B 55, 3046 (1997).

[15] S. Redner, J. Statist. Phys. 25, 15 (1981).

[16] R. Mejdani, A. Lambros, Phys. Status Solidi B 196, 433 (1996).

[17] V.V. Hovhannisyan, N.S. Ananikian, Phys. Lett. A 372, 3363 (2008).

[18] J. Kiššová, J. Strečka, Acta Phys. Pol. A 113, 445 (2008).

[19] Y. Qi, A. Du, Y. Ma, Phys. Lett. A 377 , 27 (2012).

[20] J. Strečka, F. Michaud, F. Mila, Acta Phys. Pol. A 126, 24 (2014).

[21] Y. Qi, S.-W. Lv, A. Du, N.-S. Yu, Chin. Phys. B 25, 117501 (2016).

[22] O. Rojas, J. Strečka, S.M. de Souza, Solid State Commun. 246, 68 (2016).

[23] H.A. Zad, N. Ananikian, R. Kenna, J. Phys. Condens. Matter 31, 445802 (2019).

[24] Z.-Y. Gao, D. Lv, W. Wang, J. Yu, Polymer 223, 123678 (2021).

[25] M. Yang, W. Wang, B.-C. Li, H.-J. Wu, S.Q. Yang, J. Yang, Physica A 539, 122932 (2020).

[26] E. Albayrak, Mod. Phys. Lett. B 35, 2150286 (2021).

[27] E. Albayrak, Physica B 594, 412353 (2020).

[28] E. Albayrak, Chin. J. Phys. 56, 622 (2018).

[29] E. Albayrak, Commun. Theor. Phys. 68, 361 (2017).

[30] J.A. Cuesta, A. Sánchez, J. Statist. Phys. 115, 869 (2004).

[31] J. Strečka, K. Karl'ová, O. Krupnitska, Physica E 133, 114805 (2021).

[32] F.J. Dyson, Commun. Math. Phys. 12, 91 (1969). 